\title{
Sustainable Environmental Economic Dispatch Optimization with Hybrid Metaheuristic Modification
}

\author{
M.R.M. Ridzuan, E.E. Hassan, A.R. Abdullah, A.F.A. Kadir \\ Faculty of Electrical Engineering, Universiti Teknikal Malaysia Melaka, Hang Tuah Jaya, Durian Tunggal, 76100, \\ Malaysia
}

\begin{abstract}
Article Info
Article history:

Received Jan 19, 2018

Revised Mar 23, 2018

Accepted Apr 21, 2018

Keywords:

Artificial Intelligence

Economic Dispatch

Multi Objective Function

ABSTRACT

Today's Economic Dispatch (ED) solutions are featured with environmental obligations. Hence, the significant objective functions contribute to cost minimization, lower emission and less total system losses. As an alternative, New Meta Heuristic Evolutionary Programming (NMEP) technique was proposed to optimize the individual ED problem categorized as Single Objective Environmental Economic Dispatch (SOEELD), developed from an integration of original Meta Heuristic Evolutionary Programming (Meta-EP) with Artificial Immune System (AIS) with new arrangement in the mutation and cloning processes. The comparative analysis was conducted between the original Meta-EP and classical method of Hadi Saadat to verify the performance of NMEP method. Each particular objective function identified the best possible outcomes through the NMEP method. The simulations were conducted using MATLAB programming which tested both standard IEEE 26 and 57 bus systems.
\end{abstract}

Copyright (C) 2018Institute of Advanced Engineering and Science. All rights reserved.

\section{Corresponding Author:}

M.R.M. Ridzuan,

Faculty of Electrical Engineering, Universiti Teknikal Malaysia Melaka, Hang Tuah Jaya, Durian Tunggal, 76100, Malaysia.

Email: mohamadradzi1992@gmail.com

\section{INTRODUCTION}

Power system optimization is a vital study for an optimal power operation to provide smooth and sustainable load demand [1]. The rise of energy demand and insufficient of energy resources are required for quality and secured dispatch [2]. A well-coordinated and optimized power system operation help in satisfying Economic Dispatch (ED) among users of power networks. Hence, studies need to be conducted in order to analyze and develop new tools so that the optimization issues in ED could be overcome.

Basically, the principal objective of load dispatch is to minimize the total fuel cost while satisfying the requirements of some important operational parameters. In today's environment, efficient load dispatch requires not only to schedule the power generation at the least cost but also to consider other performance factors to be optimized in power flow over the networks. The obligation of social attentions has influenced the reduction of energy conservation and pollution emission produced by power plants [3]. Hence, the total cost function alone is no longer suitable as the main focus in optimizing the ED problems. In order to reduce pollution as a result of electrical power generation, minimization on emission should be added to the objective function of ED which is generation cost minimization [4]. However, ED problems are also subject to the operational constraints and security criteria of a power system so that the secured and economic loads are dispatched equally.

Power system operation is getting more challenging due to the large number of variables working together with uncertain parameters so, the mathematical solutions for it is becoming more complicated [5]. Solutions to power system problems often involve solving optimization problems in which objective and 
constraint formulations are non-differentiable and resulted in nonlinear solutions. Thus, many studies have been conducted to overcome complicated optimization problem in power system operation.

Over the last 20 years, most optimization techniques have been categorized into three different categories namely conventional methods, intelligent searches and fuzzy set application [6]. As reported in a study [7], the Gradient based conventional approaches such as Newton Methods, linear programming and quadratic programming may result in poor solutions solving problems which are non-convex, non-continuous and have highly non-linear solutions. Alternatively, the meta-heuristic approaches are introduced aiming to optimize their chosen objective functions, hence providing globally optimal solutions [8]. Recently, new technique based on immunity algorithm, namely the Artificial Immune System (AIS) has been implemented for solving ED problems in order to minimize the fuel cost generation with consideration of some constraints [9]. Therefore, recent studies are inspired to merge conventional methods and advanced optimization techniques for better and faster optimization approaches.

This study intended to introduce a new heuristic algorithm which was an improvement to the Meta Heuristic Evolutionary Programming (NMEP) technique. The proposed technique was implemented to solve economic and environmentally constrained problems utilizing single objective function. In addition, the performances of the newly developed technique were compared with that provided by the Meta-EP and AIS along with Base technique. The best solutions were identified based on the minimum total generation cost, least total pollution and smallest total system losses.

\section{RESEARCH METHOD}

\subsection{Economic Dispatch}

\subsubsection{Objective Function}

The overall research methodology involved in the Environmental Economic Load Dispatch (EELD) was classified into four stages. The first task was to achieve the objective of the study which was to establish a new technique particularly to solve EELD optimization problem. The research approach was to design a new optimization technique taking some inspiration from the Meta-EP mutation strategy. The development also included identifying suitable objective functions which were significant to EELD problem along with some constraints as discussed in the following section [10]. In order to achieve the research objective, the development of the new single objective technique was to be accomplished. The performance of the developed technique was evaluated and compared with other techniques namely the AIS and Meta-EP along with Base technique. The developed techniques were tested on the standard IEEE 26 and 57 bus system in order to minimize the total fuel cost, emission dispersed and system losses.

\subsubsection{Total Generation Cost Minimization}

Principally, an important objective function of ED was to obtain the minimum entire cost during power system operation identified to be a total generation cost minimization. This objective function is presented in mathematical formulation as in equation (1).

$$
\begin{aligned}
& C_{i}\left(P_{g i}\right)=\alpha_{i}+b_{i} P_{g i}+c_{i} P_{g i}^{2} \\
& C_{\text {Total }}=\sum_{i=1}^{N_{g}} C_{i}\left(P_{g i}\right) \text { dollar per hour }(\$ / h)
\end{aligned}
$$

Where, $C_{i}\left(P_{g i}\right)$ is the cost of generation for unit $i, P_{g i}$ is the power generated by unit $i, \alpha_{i}, b_{i}, c_{i}$ are the cost coefficient for the unit $i$, and $C_{\text {Total }}$ is the sum function of each generating unit of $N_{g}$.

\subsubsection{Total Emission Minimization}

The next essential objective function was a total emission reduction which was dispersed by thermal generator as given by equation (2).

$$
E_{\text {Total }}=\sum_{i=1}^{N_{g}}\left(\gamma_{i} P_{g i}^{2}+\beta_{i} P_{i}+\alpha_{i}\right) \times\left(10^{-2}\right)+\varepsilon_{i} \exp \left(\lambda_{i} P_{g i}\right) \text { ton per hour }(\text { ton } / h)
$$

Where, $E_{\text {Total }}$ is the sum function for each generating emission unit of $N_{g}, \gamma_{i}, \beta_{i}, \alpha_{i}, \varepsilon_{i}, \lambda_{i}$ are the emission coefficient for the unit $i$, and $P_{g i}$ is the power generated by unit $i$. 


\subsubsection{Total System Loss Minimization}

Another significant objective function was to identify the total system loss minimization. This objective function is formulated as in equation (3).

$$
P_{\text {loss }}=\sum_{i=1}^{N_{g}} P_{\text {gi }}-P_{\text {load }} \text { Megawatt (MW) }
$$

Where, $P_{\text {loss }}$ is the sum function of each generating unit $N_{g}, P_{g i}$ is the power generated by unit $i$, and $P_{l o a d}$ is the real power load demand by unit $i$.

\subsubsection{Constraints}

In obtaining the results for whole objective function, the following equality and inequality operational constraints must be under their limitations using equations (4) and (5).

\subsubsection{Equality Constraint Formula}

$$
\sum_{i=1}^{N_{g}} P_{g i}=P_{\text {load }}+P_{\text {loss }} \operatorname{Megawatt}(M W)
$$

Where, $P_{\text {load }}$ is system load demand and $T_{\text {loss }}$ is total system losses.

\subsubsection{Inequality Constraint Formula}

$$
P_{\min } \leq P_{g i} \leq P_{\max } \text { Megawatt }(M W)
$$

Where, $P_{\min }$ is the minimum real power generation of unit, $I$ and $P_{\min }$ is the maximum real power generation of unit $i$.

\subsection{Methodology}

\section{Development of New Meta Heuristic Evolutionary Programming Technique (NMEP)}

The fundamental of NMEP was a combination between Meta-EP and AIS techniques with some algorithm modification to improve the original technique while producing a better solution for EELD problem in power system. The differences from other techniques included the modification occurring in the Gaussian mutation process and the cloning process in order to minimize the total generation cost, emission and system losses. Every technique would be simulated through the same common parameter shown in Table 1 and the results were compared to determine the best solution for the economic dispatch problem [11]. This technique was conducted in the laboratory using MATLAB simulation based on standard IEEE 26 and 57 bus system.

The single objective function involved six and seven control generator units in order to optimize the results of the single objective functions for standard IEEE 26 and 57 bus system respectively. Naturally, the main processes of NMEP are initialization, fitness, mutation, cloning and selection process to obtain the results [12]. However, some minor processes would be added to improve the results of the technique in the initialization, Gaussian mutation and cloning process which would make this technique rare from the rests. The flow chart of the whole process of NMEP technique is shown in Figure 1. The main and additional process are discussed in details below.

Initialization process was a primary process in NMEP. In this optimization, the NMEP started with some random numbers of parameter set. An important process of NMEP was selection population. The speed of optimization depended on the number of solutions in the population. In this simulation, the sets of samples used were 20 random numbers, and then the random numbers were generated representing the reactive power to be dispatched by generator in power system. Five generating units namely Pg1, Pg2, Pg3, Pg4, Pg5, Pg6, Pg8, Pg9, Pg 12 and Pg26 as the actual generators such as 1, 2, 3, 4, 5, 6, 8, 9, 12 and 26 respectively generated reactive power according to standard IEEE 26 and 57 bus system for this study. In the constraints part at the initial, stage of the process, some constraints were set to make NMEP generated only random numbers that satisfied predetermined conditions but the values must be less than the initial NMEP values. These conditions were to improve the fitness. The compatible NMEP maximum and NMEP minimum values were set not greater than 1.05 p.u. and not less than 0.95 p.u. for bus voltage limit after conducting several 
tests [13]. This condition ensures that any violation of the system can be avoided along with an improvement in the voltage profile.

The evaluation process was the first process to produce the results of fitness or known as single objective function. From the simulation, the fitness was to replicate original population to new populations. Load flow programmer was conducted to calculate the fitness. In order to complete the fitness process, the load flow programmer from the main NMEP programmer had to be called to get the results of fitness. The fitness was calculated according to equations (1) to (3).

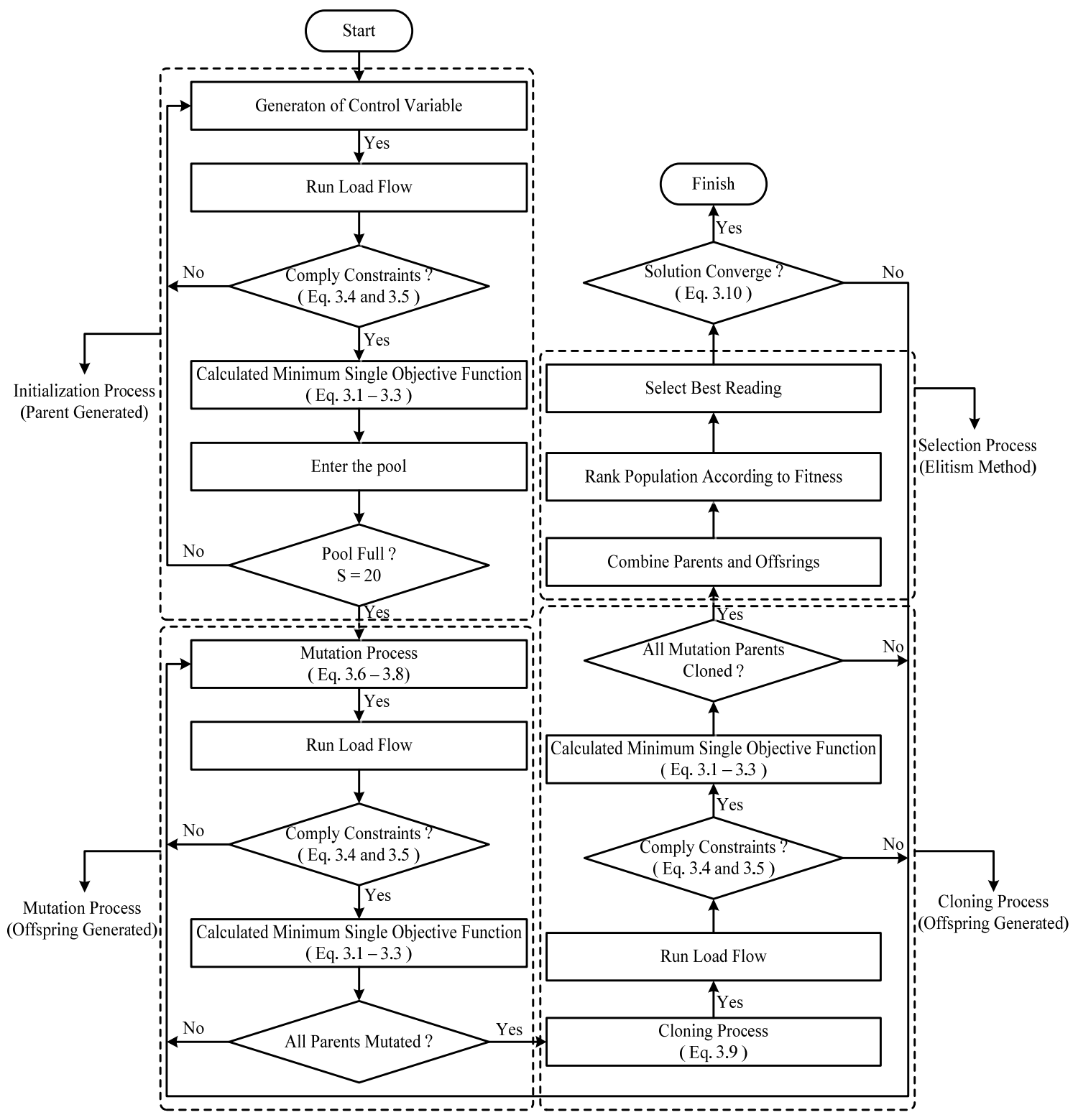

Figure 1. The Flow Chart of Multi Objective Function a NMEP Technique

Then, the mutation process was an important process for this paper because this process differed from other techniques that conducted some modifications on the programing of the Gaussian mutation process. The mutation process was producing new generation or known as offspring using equations (6) to $(8)$.

$$
\eta_{i, j}^{\prime}=\eta_{i, j} \exp \left(\tau^{\prime} N(0,1)+\tau N_{j}(0,1)\right)
$$




$$
\begin{aligned}
& L_{i, j}^{\prime}=L_{i, j}+\eta_{i, j}^{\prime}\left(N_{j}(0,1)\right) \\
& L_{o i, j}^{\prime}=L_{o i, j}+\eta_{i, j}^{\prime}\left(N_{j}(0,1)\right)
\end{aligned}
$$

Where,

$$
\begin{aligned}
\tau & =\sqrt{\frac{1}{\sqrt{2 n}}} \\
\tau^{\prime} & =\frac{1}{\sqrt{2 n}}
\end{aligned}
$$

where, $L_{i}$ and $L_{o i}, \eta_{i, j}$ and $\eta_{i, j}^{\prime}$ is the $i^{\text {th }}$ components of the respective vectors, $N(0,1)$ is the normal distribution of one dimensional random number with mean 0 and 1 and $N_{j}(0,1)$ indicates the new random number for each value of $j$.

Other than mutation process, the clonal selection algorithm reproduces those individuals with higher affinity and selected their improved matured offsprings, where single members would be locally optimized and newcomers yielded a broader exploration of the search space. This characteristic made the clonal selection algorithm suitable for solving multi-modal optimization problems. The effect of varying the number of clones generated according to the fitness (affinity) of the individual was investigated in this study. The cloning is executed in MATLAB programming using equation (9).

$$
\text { Clone }=\operatorname{repmat}(A,[a, b])
$$

Moreover, the selection of random numbers from a combination of fitness and offspring in order to identify the new generation is essential to produce the optimization value for objective function result. The population will be ranked in ascending order from the minimum to maximum optimization values. This ranking only covered 20 random samples of fitness and offspring because the new generation produced was based on the original number of samples.

In the meantime, convergence test was conducted to determine the stopping criteria of the optimization process. The convergence criterion was specified by the difference between the maximum and minimum fitness to be less than 0.0001 . If the convergence condition was not satisfied, the mutation, tournament and selection process would be repeated until convergence criterion was met using equation (10).

\begin{tabular}{|c|c|c|c|c|c|c|c|c|c|c|}
\hline \multirow{3}{*}{$\begin{array}{c}\text { No. of } \\
\text { Generato } \\
\text { r }\end{array}$} & \multicolumn{9}{|c|}{ Standard IEEE 57 Bus System } & \multirow[b]{3}{*}{$\lambda$} \\
\hline & \multicolumn{3}{|c|}{ Cost Coefficients (p.u.) } & \multicolumn{2}{|c|}{$\begin{array}{l}\text { Generator Limit } \\
\text { (MW) }\end{array}$} & & \multicolumn{3}{|c|}{ Emission coefficients (p.u.) } & \\
\hline & $a_{i}$ & $b_{i}$ & $f_{i}$ & Min & Max & $\alpha$ & $\beta$ & $\gamma$ & $\varepsilon$ & \\
\hline 1 & 115 & 2.00 & 0.0055 & 50 & 576 & 4.091 & -5.543 & 6.490 & $2.0 \mathrm{e}-4$ & 2.857 \\
\hline 2 & 40 & 3.50 & 0.0060 & 10 & 100 & 2.543 & -6.047 & 5.638 & $5.0 \mathrm{e}-4$ & 3.333 \\
\hline 3 & 122 & 3.15 & 0.0050 & 20 & 140 & 4.258 & -5.094 & 4.586 & $1.0 \mathrm{e}-6$ & 8.000 \\
\hline 6 & 125 & 3.05 & 0.0050 & 10 & 100 & 5.326 & -3.550 & 3.380 & $2.0 \mathrm{e}-3$ & 2.000 \\
\hline 8 & 120 & 2.75 & 0.0070 & 40 & 550 & 4.258 & -5.094 & 4.586 & $1.0 \mathrm{e}-6$ & 8.000 \\
\hline 9 & 70 & 3.45 & 0.0070 & 10 & 100 & 6.131 & -5.555 & 5.151 & $1.0 \mathrm{e}-5$ & 6.667 \\
\hline 12 & 150 & 1.89 & 0.0050 & 30 & 410 & 4.258 & -5.094 & 4.586 & $1.0 \mathrm{e}-6$ & 8.000 \\
\hline
\end{tabular}

$$
\text { maximum }_{\text {fitness }}-\text { minimum }_{\text {fitness }} \leq 0.0001
$$

Table 1. The Parameter Used To Produce the Result for Standard IEEE 26 and 57 Bus System

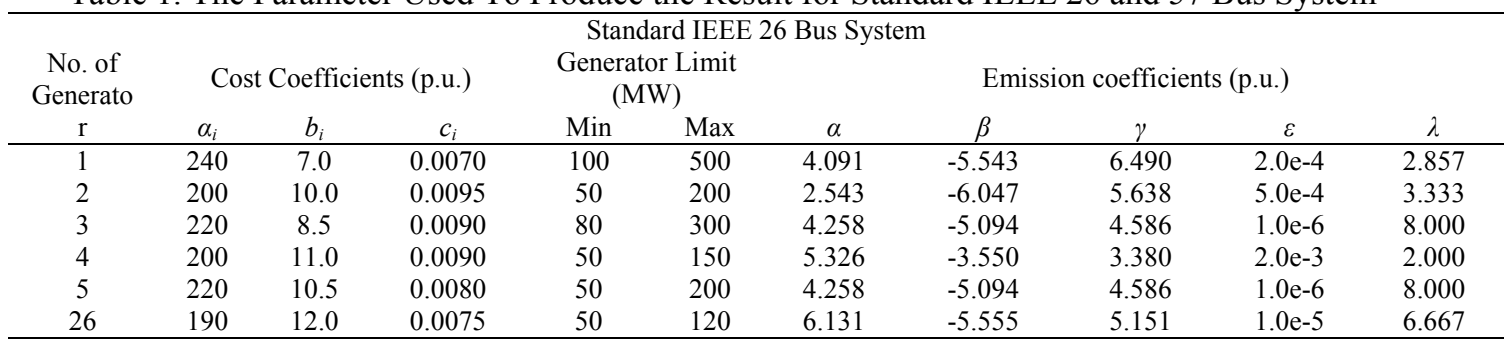




\section{RESULTS AND ANALYSIS}

The development of a New Meta Heuristic Evolutionary Programming (NMEP) algorithm was tested on standard IEEE 26 and IEEE 57 bus system using MATLAB simulation environment. The results for single objective were considered in three different categories as follows:

i) SOCEELD = total cost minimization (fitness) while total emission and total system loss (observed)

ii) SOEEELD = total emission minimization (fitness) while total cost and total system loss (observed)

iii) SOLEELD = total system loss minimization (fitness) while total cost and total emission (observed)

This particular solution highlighted the results obtained from individual objective functions (fitness) while observing the other two functions. Each solution presents the achievement for single objective function that is executed 20 times using identical optimization model as a performance measurement [14]. The results were discussed in details in term of parameter application, the best possible answers between objective functions and the overall optimal solutions were determined through the optimize finest value of objective function. In addition, the AIS, Meta-EP and Base techniques were compared to verify the quality of the performance proposed techniques solutions. However, the Base technique based on Hadi Saadat is only shown as fundamental results for both standard IEEE 26 and 57 bus systems [15].

Table 2. The Optimal Generating Units for MOCEEELD among the Three Techniques Using Fixed and Random Weights Values on Standard IEEE 26 and 57 bus

\begin{tabular}{|c|c|c|c|c|c|c|c|c|c|}
\hline \multirow[b]{2}{*}{ 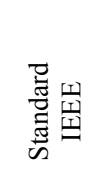 } & \multicolumn{9}{|c|}{ SOEELD } \\
\hline & 导 & $\stackrel{\substack{0 \\
a}}{\sum}$ & 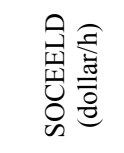 & 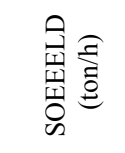 & 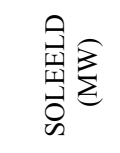 & 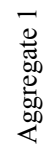 & 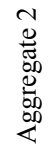 & 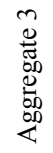 & 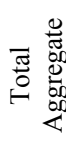 \\
\hline 26 Bus & Base & 1263.0 & 15486.19 & 19733.95 & 13.02838 & 4 & 4 & 4 & 12 \\
\hline \multirow[t]{3}{*}{ System } & AIS & 1263.0 & 15461.30 & 19251.01 & 12.99233 & 3 & 2 & 3 & 8 \\
\hline & Meta-EP & 1263.0 & 15459.30 & 19562.67 & 12.96021 & 2 & 3 & 2 & 7 \\
\hline & NMEP & 1263.0 & 15361.53 & 19063.08 & 12.86571 & 1 & 1 & 1 & 3 \\
\hline 57 Bus & Base & 1250.8 & 6493.75 & 21748.59 & 36.13481 & 4 & 4 & 4 & 12 \\
\hline \multirow[t]{3}{*}{ System } & AIS & 1250.8 & 6490.09 & 21481.10 & 34.13347 & 3 & 2 & 2 & 7 \\
\hline & Meta-EP & 1250.8 & 6487.26 & 21709.62 & 35.39216 & 2 & 3 & 3 & 8 \\
\hline & NMEP & 1250.8 & 6475.06 & 20740.36 & 34.07389 & 1 & 1 & 1 & 3 \\
\hline
\end{tabular}

The NMEP for SOCEELD provided the solution to reduce the total generation cost by not only focusing on the fuel generator but also including the other maintenance cost. Based on Table 2, the lowest entire cost was at only 15361.53 (dollar/h) using NMEP implementation. In other words, the NMEP method spent about 873985.20 (dollar/year) while 856465.20 (dollar/year) less than AIS and Meta-EP respectively. Additionally, the NMEP also showed the outperform solution as compared with AIS and Meta-EP technique as saving the profit on total generation cost about 131662.80 (dollar/year) from AIS while 106872.00 (dollar/year) than Meta-EP on 57 bus system. Hence, the NMEP was supposedly saving the average profit about RM 3357073.78 per year on 26 bus system and RM 462757.51 per year for 57 bus system based on the current currency rates ( $1 \$=\mathrm{RM} 3.88)$. The second necessary objective function namely SOEEELD was to obtain the least possible total emission dispersed through the environment caused by the operation in the power system network. According to [16] and [17], 1 ton of emission of coal is equal to the losses of 980 $\mathrm{kWh}$. Consequently, if the NMEP produced the emission about $2.95 \mathrm{TWh}$ per year, hence, the average saving on the losses of electricity was equivalent to RM 130143988.40 (if every kWh is charge at 26 cent) per year for the 26 bus system. Similarly for 57 bus system, NMEP was also able to reduce the average cost about RM 323694176.40 per year from 7.34 TWh unwanted emission dispersed per year. The following objective function was minimizing total system loss or SOLEELD in solving EELD problem. For that reason, selecting suitable generating units was a priority to achieve this particular objective. As mentioned previously, the proper generating units influenced the best possible solution. Therefore, the sum of optimal generating units which contributed to achieve the best possible SOLSEELD solutions among all mentioned optimization techniques was calculated and must be equal to $\mathrm{P}_{\text {load }}$ after adding the $\mathrm{P}_{\text {loss. }}$ From the results obtained, the smallest system loss was about 12.86571 (MW) using NMEP technique. Even though, the losses gained through AIS and Meta-EP were not obviously differed from the proposed method, about $827.82 \mathrm{MW}$ and 1109.19 MW will be lost throughout a year. Furthermore, assuming industrial tariff in Malaysia is at average cost about $44.10 \mathrm{sen} / \mathrm{kWh}$ thus, the NMEP technique save about RM 42711.07 per year for 26 bus system 
whereas RM 266142.75 per year on 57 bus system if losses capture was considered according per hour in order to get equivalent results as the comparison results.

In order to define the best performance of single objective functions in solving EELD problems, Table 2 was used to show the finest result of all identified single objective functions among those three techniques along with Base technique for both standard IEEE 26 bus system and 57 bus system. Thoroughly, the NMEP approach was compared between two other common optimization techniques known as AIS and Meta-EP methods and also with the base values as recommended by Hadi Saadat. All results were evaluated using the aggregate functions approach in order to approve the best performance solution among them. This implementation was measured by declaring the first winner with the smallest aggregate value among the comparative techniques. Based on the table, the minimum overall cost (SOCEELD), the smallest emission amount (SOEEELD) and total loss of the system (SOLEELD) were represented by Aggregate 1, Aggregate 2 and Aggregate 3 respectively. Overall, the NMEP method won the first place for Aggregate 1, Aggregate 2 and Aggregate 3 for both standard IEEE 26 and 57 bus systems. Moreover, the total aggregate column also showed the lowest value that resulted in the proposed method as the excellent technique among those three optimization techniques. In addition, the NMEP also had the fewer amounts in the identified observation quantities during the single objective solution. In short, NMEP is the most suitable technique particularly in resolving the SOEELD issues among other two common techniques in terms of the total generation cost minimization, least emission production and smallest system losses for both standard IEEE 26 and 57 bus systems.

\section{CONCLUSION}

In conclusion, the growth of energy demand and inadequacy of energy resource are required for a secured load dispatch. Nevertheless, the pressure from public awareness contributes to the requirement for reduction in toxic waste emission produced by the power plants. Thus the development of new optimization technique namely NMEP is aiming for economical load without compromising the well-being of the environment. All recognized single objective solutions are compared among the basic Meta-EP and classical method by Hadi Saadat respectively. The best possible solution for these individual objective EELD problems is obtained by the NMEP method. Therefore, the NMEP method is highly recommended particularly in solving Environmental Economic Load Dispatch problems.

\section{ACKNOWLEDGMENT}

The research is financially supported by the team of this project from Advance Digital Signal Processing Laboratory (ADSP Lab). Special thanks also go to the Faculty of Electrical Engineering of Universiti Teknikal Malaysia Melaka (UTeM) and Ministry of Higher Education Malaysia (MOHE) for giving the cooperation and funding to ensure the feasibility and success of this research namely RAGS/1/2015/TK0/FKE/03/B0094. Their support is gratefully acknowledged.

\section{REFERENCES}

[1] Li, C., De Bosio, F., Chen, F., Chaudhary, S.K., Vasquez, J.C., \& Guerrero, J.M. "Economic Dispatch for Operating Cost Minimization Under Real-Time Pricing in Droop-Controlled DC Microgrid". IEEE Journal of Emerging and Selected Topics in Power Electronics. 2017; 5(1): 587-595.

[2] Muhtazaruddin, M.N.B., Bani, N.A., Aris, S.A.M., Kaidi, H.M., Fatah, A.Y.A., Jamia, J.J., Muhammad-Sukki, F. and Abu-Bakar, S.H. "Distribution power loss minimization via distributed generation, capacitor and network reconfiguration". Indonesian Journal of Electrical Engineering and Computer Science. 2017; 5(3): 488-495.

[3] Arriffin, A.M., Othman, M.M., Kamaruzaman, A.A.M., Musirin, I., Yahya, A., \& Latip, M.F.A. "Stochastic Approach of Voltage Optimization to Maximize Power Saving in a Building". Indonesian Journal of Electrical Engineering and Computer Science. 2017; 8(1): 268-272.

[4] Qu, B.Y., Zhu, Y.S., Jiao, Y.C., Wu, M.Y., Suganthan, P.N., \& Liang, J.J. "A survey on multi-objective evolutionary algorithms for the solution of the environmental/economic dispatch problems". Swarm and Evolutionary Computation. 2018; 38: 1-11.

[5] Pourbabak, H., Luo, J., Chen, T., \& Su, W. "A novel consensus-based distributed algorithm for economic dispatch based on local estimation of power mismatch". IEEE Transactions on Smart Grid. 2017.

[6] Hajiabbas, M.P., Nazari-Heris, M., Madadi, S., \& Mohammadi-Ivatloo, B. "The utilization of quantum inspired computational intelligent in power systems optimization". In Quantum Computing: An Environment for Intelligent Large Scale Real Application.2018: 489-505.

[7] Du, D., Wang, C., Du, X., Yan, S., Ren, X., Shi, X., \& Hein, J. R. "Distance-gradient-based variogram and Kriging to evaluate cobalt-rich crust deposits on seamounts". Ore Geology Reviews. 2017; 84: 218-227.

[8] Pattanaik, J.K., Basu, M., \& Dash, D.P. "Review on application and comparison of metaheuristic techniques to multi-area economic dispatch problem". Protection and Control of Modern Power Systems. 2017; 2(1): 17.

Sustainable Environmental Economic Dispatch Optimization with Hybrid Metaheuristic ... (M.R.M. Ridzuan) 
[9] Yang, Z., Xiang, J., \& Li, Y. "Distributed consensus based supply-demand balance algorithm for economic dispatch problem in a smart grid with switching graph". IEEE Transactions on Industrial Electronics. 2017; 64(2): 1600-1610.

[10] Ajenikoko, G.A., \& Olabode, O.E. "Optimal Power Flow with Reactive Power Compensation for Cost and Loss Minimization on Nigerian Power Grid System". Indonesian Journal of Electrical Engineering and Informatics (IJEEI). 2017; 5(3): 236-247.

[11] Ridzuan, M.R.M., Hassan, E.E., Abdullah, A.R., Bahaman, N., \& Kadir, A.F.A. "NMEP based Gaussian Mutation Process on Optimizing Fitness Function for MOEED". International Journal on Advanced Science, Engineering and Information Technology. 2017; 7(5): 1840-1846.

[12] Biethahn, J., \& Nissen, V. "Evolutionary algorithms in management applications". Springer Science \& Business Media. 2012

[13] Hassan, E.E., Rahman, T.K.A., Zakaria, Z., \& Bahaman, N. "The Improved of BFOA for Ensuring the Sustainable Economic Dispatch". In Applied Mechanics and Materials. 2015: 785: 83-87

[14] Hassan, E.E., Zakaria, Z., \& Rahman, T.K.A. "Improved Adaptive Tumbling Bacterial Foraging Optimization (ATBFO) for emission constrained economic dispatch problem". In Proceedings of the World Congress on Engineering. 2012; 2: 1-4.

[15] Aryani, N.K., Soeprijanto, A., Negara, I.M.Y., \& Syai'in, M. "Economic Dispatch using Quantum Evolutionary Algorithm in Electrical Power System involving Distributed Generators". International Journal of Electrical and Computer Engineering (IJECE). 2017; 7(5): 2365.

[16] Li, R., Zhan, W., \& Hao, Z. "Artificial Immune Particle Swarm Optimization Algorithm Based on Clonal Selection". Boletín Técnico. 2017; 55(1): 158-164.

[17] Cushman-Roisin, B., Gacic, M., Poulain, P. M., \& Artegiani, A. "Physical oceanography of the Adriatic Sea: past, present and future". Springer Science \& Business Media. 2013. 